\title{
SIMULATIONS OF COUPLING EFFECTS IN VIBRATION OF FGM PLATES BY MESH-FREE METHODS
}

\author{
V. SLADEK, L. SATOR \& J. SLADEK \\ Institute of Construction and Architecture, Slovak Academy of Sciences, Bratislava, Slovakia.
}

\begin{abstract}
In this paper, we present briefly the derivation of the equations of motion and boundary conditions for elastic plates with functionally graded Young's modulus and mass density of the plate subjected to transversal transient dynamic loads. The unified formulation is derived for three plate bending theories, such as the Kirchhoff-Love theory (KLT) for bending of thin elastic plates and the shear deformation plate theories (the first order - FSDPT, and the third order - TSDPT). It is shown that the transversal gradation of Young's modulus gives rise to coupling between the bending and inplane deformation modes in plates subject to transversal loading even in static problems. In dynamic problems, there are also the inertial coupling terms. The influence of the gradation of material coefficients on bending and in-plane deformation modes with including coupling is studied in numerical experiments with consideration of Heaviside impact loading as well as Heaviside pulse loading. To decrease the order of the derivatives in the coupled PDE with variable coefficients, the decomposition technique is employed. The element-free strong formulation with using meshless approximations for spatial variation of field variables is developed and the discretized ordinary differential equations with respect to time variable are solved by using time stepping techniques. The attention is paid to the stability of numerical solutions. Several numerical results are presented for illustration of the coupling effects in bending of elastic FGM (Functionally Graded Material) plates. The role of the thickness and shear deformations is studied via numerical simulations by comparison of the plate response in three plate bending theories.
\end{abstract}

Keywords: functional gradations of young's modulus and mass density, MLS approximations, plate bending theories, strong formulation, transient dynamic load.

\section{INTRODUCTION}

The FGM composites have gained significant utilization in design of structural elements not only because of superior properties of micro-constituents but also for elimination of interface discontinuities occurring in laminated composite structures. FGMs became very attractive in a wide variety of plate problems (static, dynamic, buckling,...) considered within various plate theories (KLT, SDPT, higher order plate theories, Von-Karman assumptions for geometrical nonlinearities) and loadings (mechanical, thermal, electromagnetic,...). The most frequently used modelling of functional variation of material coefficients is the rule of mixture where the material coefficients of multiphase materials are related directly to the volume fractions and individual coefficients of the constituents [1,2]. From the physical point of view as well as from the view of engineering applications the transversal gradation of material properties is more interesting than the in-plane gradation in plate problems. The transverse gradation gives rise to coupling between the deflections and in-plane deformations [3-6] even in the case of thin plate theory and static problems. Since gradations of material coefficients affect the coefficients in equations of motion for plate bending problems, one can expect an influence of the material gradation not only on the magnitude of the response to dynamic loadings but also on the frequency of such response. Therefore, a detailed study of FGM plates is topical and interesting form the view of both the physical and mathematical aspects in both the static [3-6] and dynamic loading conditions. In recent 
work [7], we have studied the coupling effects in thin as well as thick elastic plates subject to transient dynamic loadings via numerical simulations in plates without consideration of damping.

In this paper, we extend the formulation to FGM plates under the impact loads and pulse impact loads with including Rayleigh damping (damping is present in real engineering problems). We present briefly the derivation of the unified formulation for plate bending problems within three different theories such as the Kirchhoff-Love theory (KLT) for thin plates, the 1st order and 3rd order shear deformation plate theories (FSDPT and TSDPT). Despite the shortcomings of the KLT and the FSDPT, they are applicable to bending problems of thin and moderate thick plates, respectively, and because of certain simplifications they are still attractive. Recall that it is impossible to extend the formulation derived for a homogeneous plate to FGM plates by simple replacement of constant material coefficients by spatially variable ones. The correct derivation of equations of motion leads to discovery of coupling between the bending and in-plane deformation modes as well as to specification of necessary conditions for such a coupling. Since the governing equations are given by rather complex PDE with high order derivatives of field variables, an efficient numerical treatment has been proposed by simplifying the mathematical complexity with preserving the physical nature of the problems. In order to decrease the order of derivatives, the original governing equations are decomposed into the system of lower order PDE with introducing new field variables. Then, the strong formulation is developed with using the Moving Least Square (MLS) approximation for spatial variations of field variables. The semi-discretized governing equations lead to a system of the ordinary differential equations for nodal unknowns, which can be solved by using standard time stepping techniques. In this paper, we employ the Wilson $\theta$-method [8] which is one-step, implicit method offering unconditional stability if $\theta \geq 1.37$. In numerical simulations, we illustrate how the frequency and amplitudes of response oscillations are affected by gradation parameters, such as the levels and exponents of the power-law gradations of the Young modulus and/or mass density.

\section{UNIFIED FORMULATION FOR TRANSIENT ELASTODYNAMIC FGM COMPOSITE PLATE BENDING PROBLEMS}

Let us consider a straight plate structure occupying the 3D domain $V=\left\{\forall\left(x_{1}, x_{2}, x_{3}\right) \in \mathbb{R}^{3}\right.$; $\left.\mathbf{x}=\left(x_{1}, x_{2}\right) \in \Omega, x_{3} \in[-h / 2, h / 2]\right\}=\Omega \times[-h / 2, h / 2]$, with the thickness being significantly smaller than the in-plane dimensions, $h \ll L$. Expanding the in-plane displacements $v_{\alpha}\left(\mathbf{x}, x_{3}, t\right)$ up to $\left(x_{3}\right)^{3}$, and assuming the deflection $v_{3}\left(\mathbf{x}, x_{3}, t\right)=w(\mathbf{x}, t)$ to be independent on the transversal coordinate $x_{3}$, one can write the displacements field as

$$
v_{i}\left(\mathbf{x}, x_{3}, t\right)=\delta_{i \alpha}\left\{u_{\alpha}(\mathbf{x}, t)+\left[c_{1} \phi\left(x_{3}\right)-x_{3}\right] w_{, \alpha}(\mathbf{x}, t)+c_{1} \phi\left(x_{3}\right) \varphi_{\alpha}(\mathbf{x}, t)\right\}+\delta_{i 3} w(\mathbf{x}, t),
$$

where the five field variables $u_{\alpha}(\mathbf{x}, t), w(\mathbf{x}, t)$, and $\varphi_{\alpha}(\mathbf{x}, t)$ stand for the in-plane displacements, deflection and rotation fields, respectively, and we have used the following notations $\phi\left(x_{3}\right):=x_{3}-c_{2} \psi\left(x_{3}\right), \psi\left(x_{3}\right):=4\left(x_{3}\right)^{3} / 3 h^{2}$, with

$$
c_{1}=\left\{\begin{array}{ll}
0, & \mathrm{KLT} \\
1, & \mathrm{SDPT}
\end{array}, c_{2}=\left\{\begin{array}{lr}
0, & \text { FSDPT, } \\
1, & \mathrm{TLTT} \\
& \mathrm{TSDPT}
\end{array}\right.\right.
$$


being two key factors for switching among the three theories (KLT, FSDPT, TSDPT). Recall that the dependence of displacements on $x_{3}$ coordinate is known and hence, this is true also for strains and stresses. Thus, we have to solve $2 \mathrm{D}$ problems. As regards the functional gradation of the Young modulus and mass density, we assume the power-law gradation in the transversal direction as

$$
\begin{aligned}
& E\left(\mathbf{x}, x_{3}\right)=E_{0} E_{V}\left(x_{3}\right), E_{V}\left(x_{3}\right)=1+\zeta\left(\frac{1}{2}+\frac{x_{3}}{h}\right)^{p}, \\
& \rho\left(\mathbf{x}, x_{3}\right)=\rho_{0} \rho_{V}\left(x_{3}\right), \rho_{V}\left(x_{3}\right)=1+\eta\left(\frac{1}{2}+\frac{x_{3}}{h}\right)^{q} .
\end{aligned}
$$

It will be seen that the transversal gradation of the Young modulus gives rise to coupling between the bending and in-plane deformation modes. Therefore, it is impossible to take into account the governing equations derived for homogeneous plate and modify their coefficients with incorporating the spatial variation of material coefficients. Equations of motion as well as the boundary condition possibilities can be derived using Hamilton's principle

$$
\delta \int_{0}^{T}\left(U-W_{e}-K\right) d t=0
$$

Without going into details [7], we present the governing equations written for the primary field variables $\left\{u_{\alpha}^{*}(\mathbf{x}, t), w^{*}(\mathbf{x}, t), \varphi_{\alpha}^{*}(\mathbf{x}, t)\right\}$

$$
\begin{gathered}
C^{(u u)} \tau_{\alpha \beta, \beta}^{*(u)}(\mathbf{x}, t)+C^{(u \varphi)} \tau_{\alpha \beta, \beta}^{*(\varphi)}(\mathbf{x}, t)+C^{(u w)} \tau_{\alpha \beta c \beta}^{*(w)}(\mathbf{x}, t) \\
+D^{(u u)} \ddot{u}_{\alpha}^{*}(\mathbf{x}, t)+D^{(u \varphi)} \ddot{\varphi}_{\alpha}^{*}(\mathbf{x}, t)+D^{(u w)} \ddot{w}_{, \alpha}^{*}(\mathbf{x}, t)=0 \\
C^{(w u)} \tau_{\alpha \beta, \alpha \beta}^{*(u)}(\mathbf{x}, t)+C^{(w \varphi)} \tau_{\alpha \beta, \alpha \beta}^{*(\varphi)}(\mathbf{x}, t)+C^{(w w)} \tau_{\alpha \beta, \alpha \beta}^{*(w)}(\mathbf{x}, t) \\
+\left(\frac{L}{h_{0}}\right)^{2} C^{s}\left[w_{, \beta \beta}^{*}(\mathbf{x}, t)+\frac{L}{h_{0}} \varphi_{\beta, \beta}^{*}(\mathbf{x}, t)\right]+D_{1}^{(w w)} \ddot{w}^{*}(\mathbf{x}, t) \\
-\left[D^{(w u)} \ddot{u}_{\beta}^{*}(\mathbf{x}, t)+D^{(w \varphi)} \ddot{\varphi}_{\beta}^{*}(\mathbf{x}, t)+D_{2}^{(w w)} \ddot{w}_{, \beta}^{*}(\mathbf{x}, t)\right]_{, \beta}=-q^{*}(\mathbf{x}, t) \\
C^{(\varphi u)} \tau_{\alpha \beta, \beta}^{*(u)}(\mathbf{x}, t)+C^{(\varphi \varphi)} \tau_{\alpha \beta, \beta}^{*(\varphi)}(\mathbf{x}, t)+C^{(\varphi w)} \tau_{\alpha \beta, \beta}^{*(w)}(\mathbf{x}, t) \\
\quad-\left(\frac{L}{h_{0}}\right)^{2} C^{s}\left[w_{, \alpha}^{*}(\mathbf{x}, t)+\frac{L}{h_{0}} \varphi_{\alpha}^{*}(\mathbf{x}, t)\right] \\
+D^{(\varphi u)} \ddot{u}_{\alpha}^{*}(\mathbf{x}, t)+D^{(\varphi \varphi)} \ddot{\varphi}_{\alpha}^{*}(\mathbf{x}, t)+D^{(\varphi w)} \ddot{w}_{, \alpha}^{*}(\mathbf{x}, t)=0,
\end{gathered}
$$

in which

$$
\begin{gathered}
\tau_{a \beta}^{*(u)}(\mathbf{x}, t):=\frac{H}{2}\left(u_{\alpha, \beta}^{*}(\mathbf{x}, t)+u_{\beta, a}^{*}(\mathbf{x}, t)\right)+v \delta_{a \beta} u_{\gamma, \gamma}^{*}(\mathbf{x}, t) \\
\tau_{a \beta, \beta}^{*(u)}(\mathbf{x}, t)=\frac{H}{2} \nabla^{2} u_{a}^{*}(\mathbf{x}, t)+\frac{H+2 v}{2} u_{\beta, \beta a}^{*}(\mathbf{x}, t), \tau_{a \beta, a \beta}^{*(u)}(\mathbf{x}, t)=(H+v) \nabla^{2} u_{\beta, \beta}^{*}(\mathbf{x}, t)
\end{gathered}
$$




$$
\begin{gathered}
\tau_{a \beta}^{*(\varphi)}(\mathbf{x}, t):=\frac{H}{2}\left(\varphi_{a, \beta}^{*}(\mathbf{x}, t)+\varphi_{\beta, \alpha}^{*}(\mathbf{x}, t)\right)+v \delta_{\alpha \beta} \varphi_{\gamma, \gamma}^{*}(\mathbf{x}, t) \\
\tau_{\alpha \beta, \beta}^{*(\varphi)}(\mathbf{x}, t)=\frac{H}{2} \nabla^{2} \varphi_{a}^{*}(\mathbf{x}, t)+\frac{H+2 v}{2} \varphi_{\beta, \beta a}^{*}(\mathbf{x}, t), \tau_{\alpha \beta, \alpha \beta}^{*(\varphi)}(\mathbf{x}, t)=(H+v) \nabla^{2} \varphi_{\beta, \beta}^{*}(\mathbf{x}, t) \\
\tau_{\alpha \beta}^{*(w)}(\mathbf{x}, t):=H w_{, \alpha \beta}^{*}(\mathbf{x}, t)+v \delta_{\alpha \beta} w_{, \gamma \gamma}^{*}(\mathbf{x}, t) \\
\tau_{\alpha \beta, \beta}^{*(w)}(\mathbf{x}, t)=(H+v) \nabla^{2} w_{, \alpha}^{*}(\mathbf{x}, t), \tau_{a \beta, \alpha \beta}^{*(w)}(\mathbf{x}, t)=(H+v) \nabla^{2} \nabla^{2} w^{*}(\mathbf{x}, t) .
\end{gathered}
$$

The superscript $(*)$ is used for dimensionless quantities [7]. Taking into account the governing eqns (5)-(7) and the explicit expressions for the coefficients $C^{(\cdot)}$ and $D^{(\cdot)}$ (see e.g. [7]), one can find the necessary condition for the static coupling between the bending and in-plane deformation modes is the transversal gradation of Young's modulus (since $C^{(u \varphi)}=C^{(\varphi u)} \neq 0$ and $C^{(u w)} \neq 0, C^{(w u)} \neq 0$, if $\left.\zeta \neq 0\right)$, while the necessary condition for the dynamic coupling is the transversal gradation of the mass density ( $\operatorname{since} D^{(u \varphi)}=D^{(\varphi u)} \neq 0$ and $D^{(u w)} \neq 0, D^{(w u)} \neq 0$, if $\left.\eta \neq 0\right)$. The coupling between the deflections and rotations $(w-\varphi$ coupling) is controlled by the key factors $c_{1}$ and $c_{2}$.

In order to eliminate the 3 rd and 4th order derivatives of field variables in the governing equations, we propose to utilize the decomposition of the derived system of the PDE into a set of PDE with derivatives not higher than second order [3] by introducing new field variables $m^{*}(\mathbf{x}, t), s_{\alpha}^{*}(\mathbf{x}, t), f_{\alpha}^{*}(\mathbf{x}, t)$ as

$$
m^{*}(\mathbf{x}, t):=\nabla^{2} w^{*}(\mathbf{x}, t), s_{\alpha}^{*}(\mathbf{x}, t):=\nabla^{2} u_{\alpha}^{*}(\mathbf{x}, t), f_{\alpha}^{*}(\mathbf{x}, t):=\nabla^{2} \varphi_{\alpha}^{*}(\mathbf{x}, t) .
$$

Then, the number of field variables is doubled and the governing equations for $\left\{u_{\alpha}^{*}(\mathbf{x}, t), s_{\alpha}^{*}(\mathbf{x}, t), w^{*}(\mathbf{x}, t), m^{*}(\mathbf{x}, t), \varphi_{\alpha}^{*}(\mathbf{x}, t), f_{\alpha}^{*}(\mathbf{x}, t)\right\}$ are given by eqns (5)-(7) and (9), with

$$
\begin{gathered}
\tau_{\alpha \beta, \beta}^{*(u)}=\frac{H}{2} s_{a}^{*}+\frac{H+2 v}{2} u_{\beta, \beta a}^{*}, \tau_{a \beta, a \beta}^{*(u)}=(H+v) s_{\beta, \beta}^{*} \\
\tau_{a \beta, \beta}^{*(p)}=\frac{H}{2} f_{a}^{*}+\frac{H+2 v}{2} \varphi_{\beta, \beta a}^{*}, \tau_{a \beta, a \beta}^{*(p)}=(H+v) f_{\beta, \beta}^{*} \\
\tau_{\alpha \beta, \beta}^{*(w)}=(H+v) m_{, \alpha}^{*}, \tau_{a \beta, a \beta}^{*(w)}=(H+v) \nabla^{2} m^{*} .
\end{gathered}
$$

Recall that the formulations within three particular theories can be obtained from the presented unified formulation by proper selection of the key factors $c_{1}, c_{2}$.

\section{NUMERICAL IMPLEMENTATION}

The MLS-approximation belongs to mesh-free approximations, since no predefined connectivity among nodal points is required [9]. The nodal points are freely distributed inside the analyzed domain and on its boundary. Beside the standard MLS-approximation, one can utilize also the Central Approximation Node (CAN) concept $[10,11]$ which enables to utilize the local support character of the approximation efficiently. In the case of strong formulations, however, the advantages of the local support approximation are utilized immediately $[12,13]$. The MLS-approximation of a field variable $g(\mathbf{x}, t)$ is expressed in terms of the shape functions $\psi^{\bar{a}}(\mathbf{x})$ and certain nodal unknowns $\hat{g}^{\bar{a}}(t)$ as

$$
\left.g(\mathbf{x}, t)\right|_{\Omega^{q}}=\sum_{a=1}^{N^{q}} g^{\bar{a}}(t) \psi^{\bar{a}}(\mathbf{x}), \Omega \supset \Omega^{q} \ni \mathbf{x}^{q},
$$


where the central approximation nodal point $\mathbf{x}^{q}$ can be selected as the nearest nodal point to the point of approximation $\mathbf{x}$, and $\bar{a}:=n(q, a)$ is the global number while $a$ is the local number of the node from the support domain of the nodal point $\mathbf{x}^{q}$.

For solution of the system of the ODE for nodal unknowns

$$
u(t) \in\left\{u_{\alpha}^{* a}(t), s_{\alpha}^{* a}(t), \varphi_{\alpha}^{* a}(t), f_{\alpha}^{* a}(t), w^{* a}(t), m^{* a}(t)\right\}_{a=1}^{N},(\alpha=1,2)
$$

we employ the Wilson $\theta$-method $[8,14,15]$ which assumes a linear change of accelerations within the time interval $\left[t_{k}, t_{k}+\theta \Delta t\right]$, where $\Delta t$ is the time step and $t_{k+1}=t_{k}+\Delta t$. The proper correlation between the spatial and temporal discretization determines the stability of numerical solutions. The Courant-Friedrichs-Levy (CFL) criterion is usually used as the necessary but not sufficient condition for stability: $|c \Delta t / \delta| \leq 1$, where $c, \Delta t$ and $\delta$ are the velocity, time step and spatial discretization step, respectively. Therefore, after many time steps one can observe divergence or non-physical damping of the numerical solution of transient problems. In the case of coupled field transient problems, the proper selection of the time step becomes problematic, if spreading of excitations of particular fields is characterized by various velocities. This is expected in the considered transient problems in FGM plates, because of the coupling between the bending and in-plane deformation modes with various velocities of elastic waves.

\section{NUMERICAL EXAMPLES}

Let us consider the square plate $L \times L(L=1)$ with clamped edges and constant thickness $h=h_{0} \in\{L / 50, L / 5\}$. The plates are either homogeneous or FGM with transversally graded Young's modulus and/or mass density (see eqn. (3)). The Poisson ratio is assumed to be constant $v=0.3$. The homogeneous initial conditions are assumed for plates under transversal loading with two kinds of time dependence: (i) impact load $q^{*}(\mathbf{x}, t)=H(t)$, (ii) impact pulse load $q^{*}(\mathbf{x}, t)=H(t)-H\left(t-t_{I}\right)$, where $H(t)$ is the Heaviside unite step function and $t_{I}$ is the duration of the pulse load. Recall that the dimensionless loading is correlated with the actual loading as

$$
q^{*}(\mathbf{x}, t)=\frac{L^{4}}{D_{0} h_{0}} q(\mathbf{x}, t),
$$

hence, the actual loading is proportional to $\left(h_{0}\right)^{4}$ during $q^{*}(\mathbf{x}, t)=1$. The Rayleigh damping is added to semi-discretized equations of motion as

$$
[\hat{K}]\{v\}+[\hat{M}]\{\ddot{v}\}=\{q\} \rightarrow[\hat{K}]\{v\}+[\hat{M}]\{\ddot{v}\}+[\hat{C}]\{\dot{v}\}=\{q\}
$$

with $[\hat{C}]=\alpha[\hat{M}]+\beta[\hat{K}]$.

In the numerical computations we have used a uniform distribution of 256 nodes, i.e. the shortest distance between two neighbour nodes is $\delta=1 / 15$. The size of the time step is variable according to the velocity of spreading of elastic excitations depending on the plate thickness as well as on the bending stiffness which depends on the gradation of Young's modulus. In the MLS-approximation, we have chosen the radius of the interpolation support domain $r^{a}=3.001 \delta$, the shape parameter in Gaussian weights $c^{a}=\delta$, and cubic polynomial basis with 10 monomials. In the Wilson $\theta$-method, we have chosen $\theta=1.4$ in order to guarantee unconditional stability. 
4.1 Example 1- Plates under Heaviside impact loading

Firstly, consider the homogeneous plate subject to impact loading. Fig. 1 shows oscillations of the thin plate $(L / h=50)$ obtained by three plate bending theories. Rather small differences

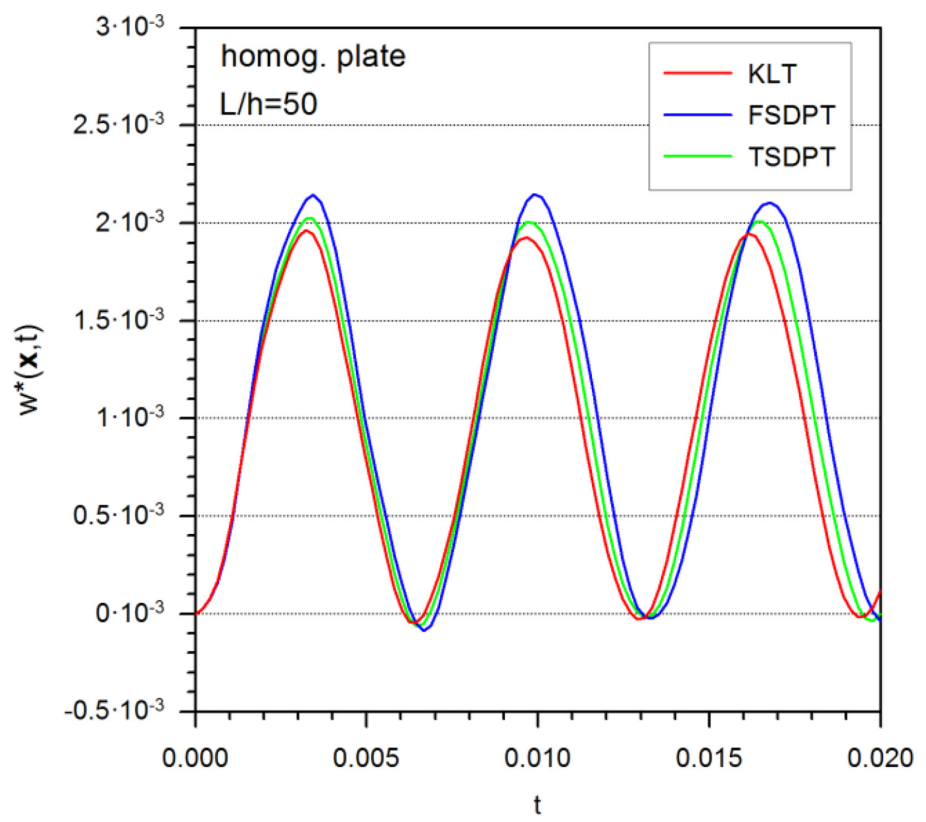

(a)

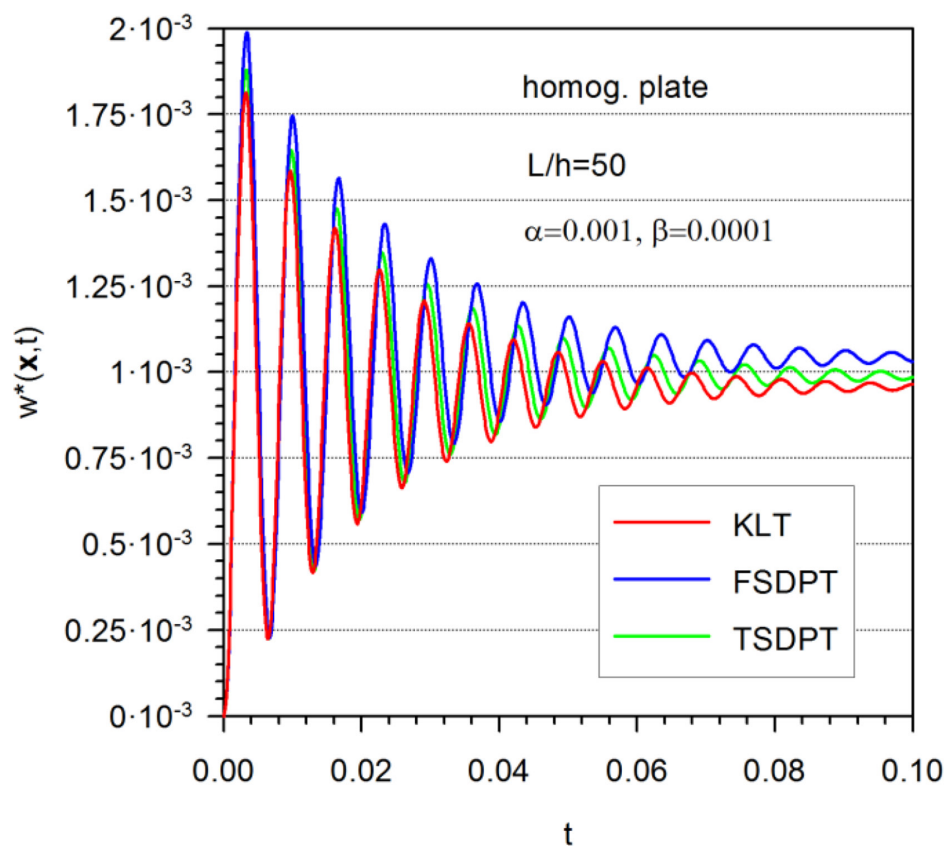

(b)

Figure 1: Deflection oscillations of a thin homogeneous plate under impact loading: (a) without damping, (b) with damping. 


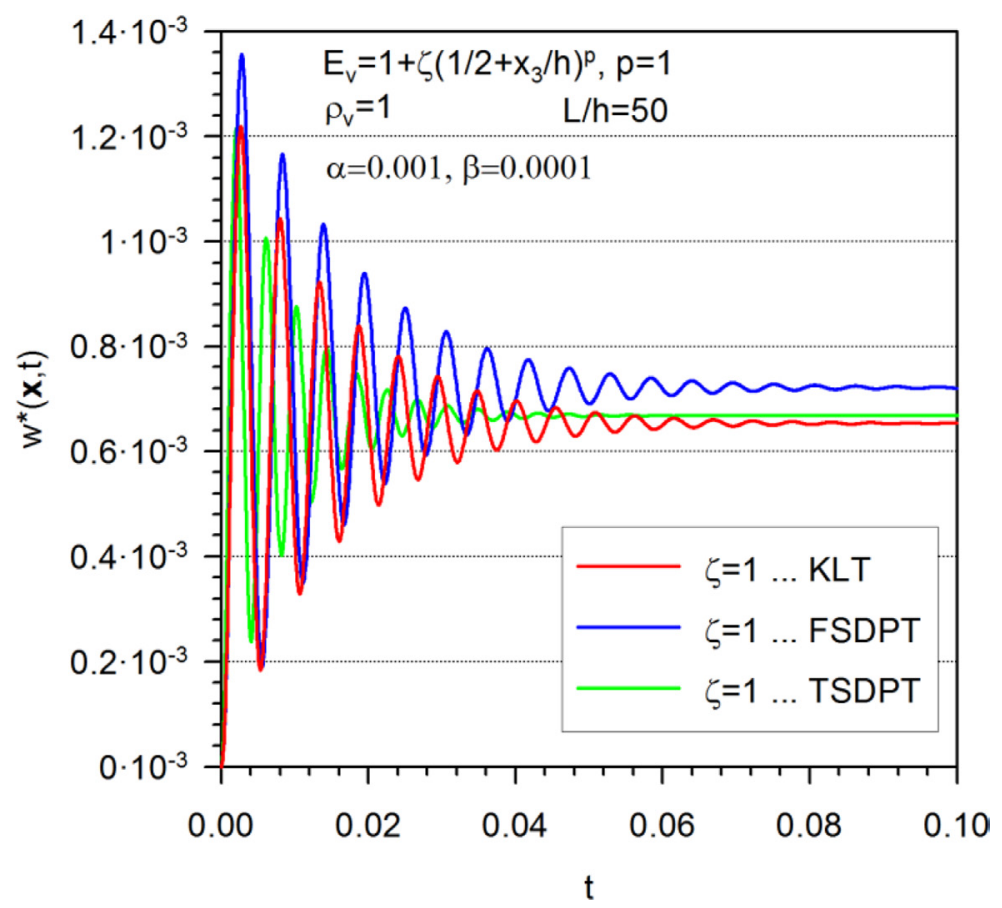

Figure 2: Deflection oscillations of a thin FGM plate under impact loading.

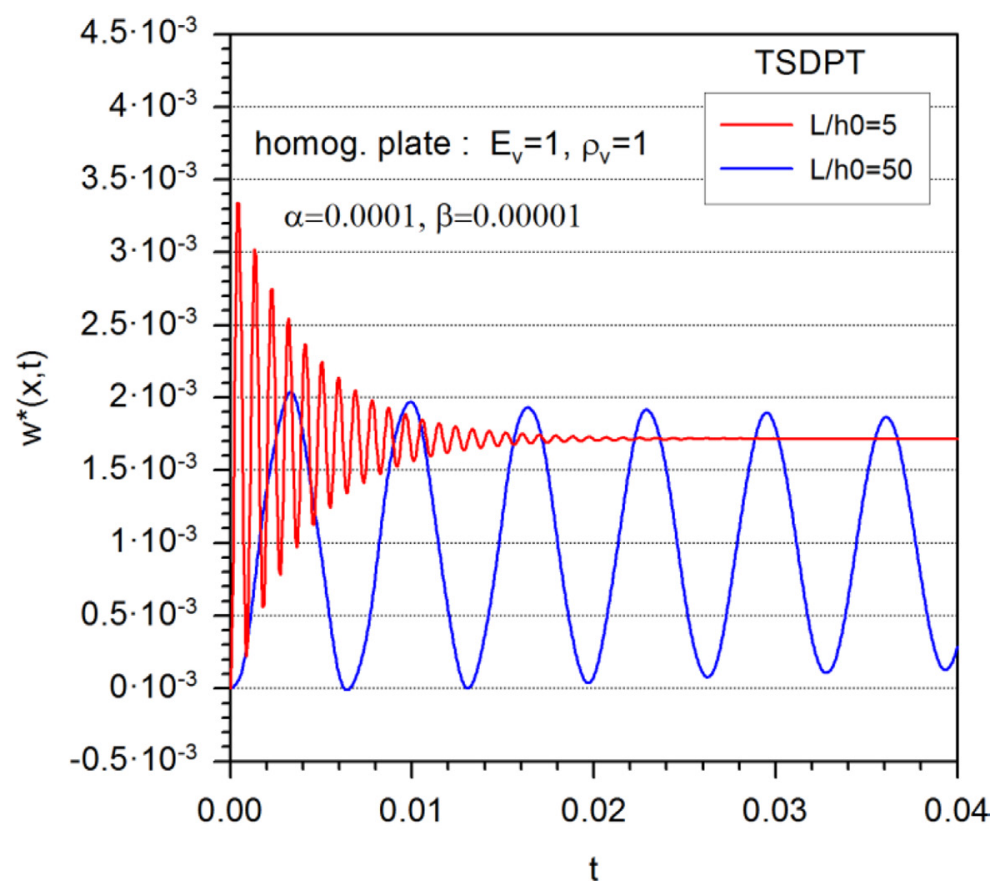

Figure 3: Deflection oscillations of thin and thick FGM plates under impact loading with consideration of damping and using TSDPT. 

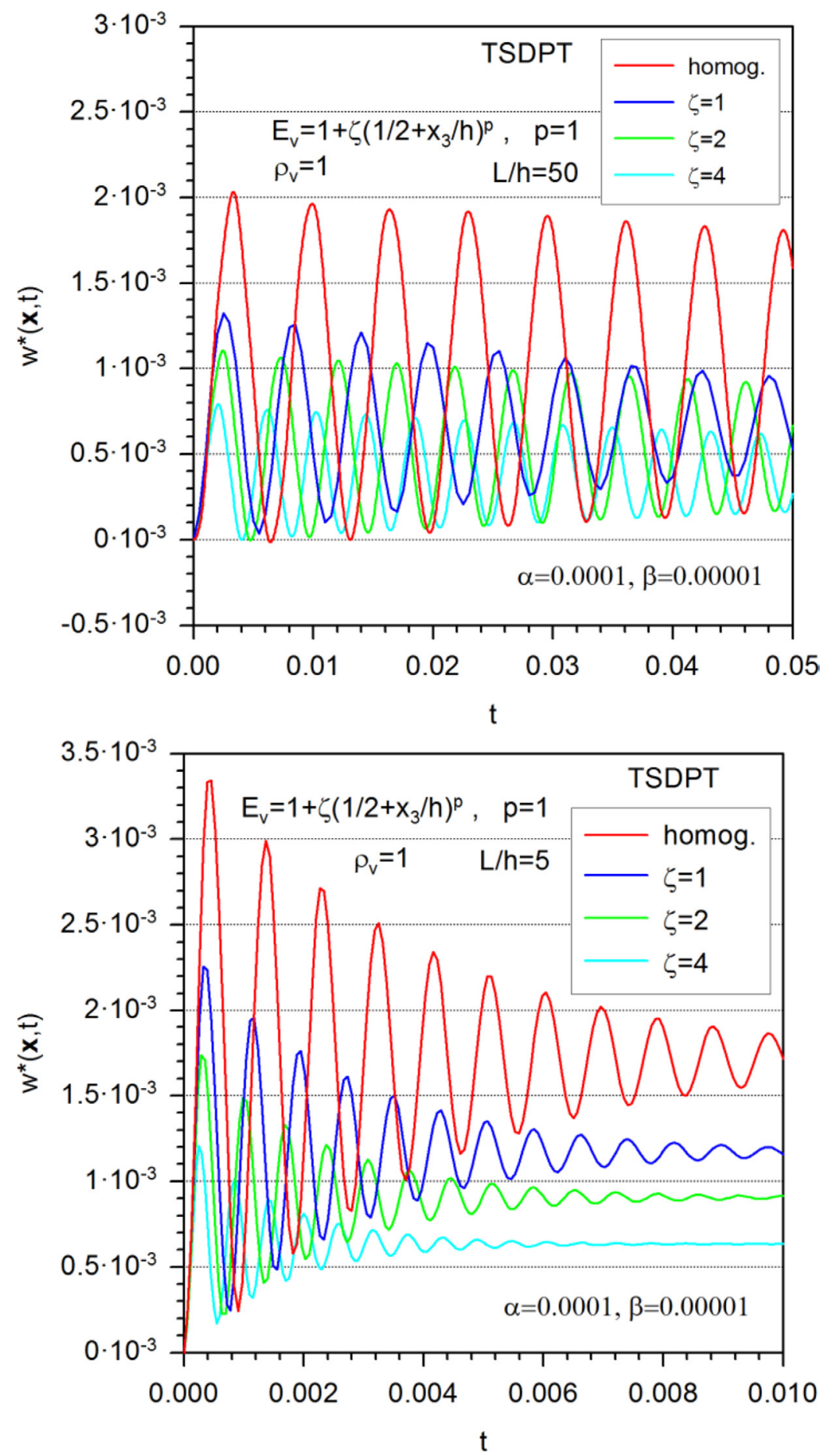

Figure 4: Deflection oscillations of thin and thick homogeneous and FGM plates under impact loading with consideration of damping and using TSDPT. 
can be seen in both the frequency and amplitude of oscillations obtained by particular theories. The deflections oscillate around the centre of oscillations with negligible damping and with unilateral bonding due to applied load. The centre of oscillations corresponds to the static value of deflection of the plate subjected to the static load $q^{*}(\mathbf{x})=1$. The negligible damping demonstrates the stability of the numerical solutions adjusted by a proper selection of the time step. The underestimation of the size of time step leads to divergent solutions, while overestimation yields non-physical damping of oscillations of the plate without consideration of damping. Note that the solutions with damping are stable for much longer time intervals than those without damping. Nevertheless the differences between the results by particular theories are increasing with increasing the time instant of observation even in the case of thin plate.

The differences in both the frequencies and amplitudes for results by three mentioned theories are more expressive in the case FGM thin plates (see Fig. 2).

Figure 3 illustrates several differences between the responses of the thin and thick homogeneous plates on the Heaviside impact load. Since the bending stiffness of the thick plate is higher than that of the thin plate, the frequency as well as the damping are higher too. Furthermore, the central value of oscillations (i.e. the value $w^{*}(\mathbf{x}, t \rightarrow \infty)$ ) as well as the first amplitude are higher for the thick plate than for the thin plate. This can be explained by the fact that we have used the same dimensionless load $q^{*}(\mathbf{x}, t)=H(t)$ for both plates and the actual load is proportional to $h^{4}$ as mentioned above.

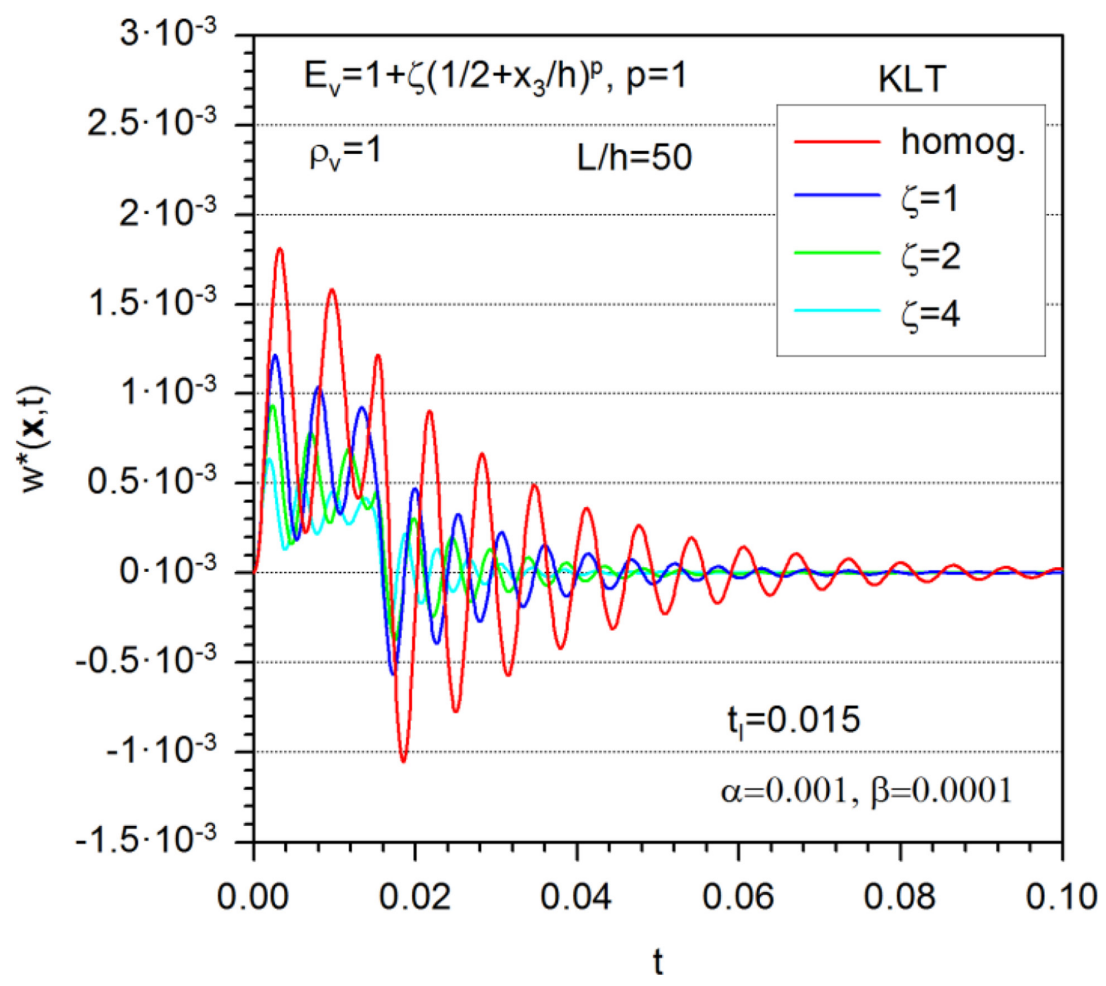

Figure 5: Deflection oscillations of thin homogeneous and FGM plates under impact pulse loading with consideration of damping and using KLT. 
Oscillations of the midpoint of the thin and thick homogeneous as well as FGM plates are shown in Fig. 4, where we have used three different levels of power-law gradation of the Young modulus.

The higher frequency and stronger damping are observed again for the thick plates than for the thin plates. Moreover, the frequency and damping are increasing, while the central values of oscillations are decreasing with increasing the level of gradation of Young's modulus in FGM plates because of increasing the bending stiffness.

Finally, Fig. 5 shows the oscillations of the mid-point deflections of thin plates under impact pulse load.

\section{CONCLUSIONS}

Transient elastodynamic problems for FGM plates are studied within three plate bending theories (KLT, FSDPT and TSDPT). The main result is the discovery of coupling between the bending and in-plane deformation modes in FGM plates with transversally graded material coefficients, such as the Young modulus and mass density.

An efficient numerical implementation has been proposed and developed for numerical solution of initial-boundary value problems for the considered multifield problems.

The great attention is paid to the numerical study of coupling effects in FGM plates subjected to transversal Heaviside impact loading and/or Heaviside pulse loading. The achieved numerical results are thoroughly discussed and interpreted.

\section{ACKNOWLEDGEMENTS}

This work was partially supported by the Slovak Research and Development Agency under the contract No. APVV-14-0440.

\section{REFERENCES}

[1] Birman, V. \& Byrd, L.W., Modeling and analysis of functionally graded materials and structures. Applied Mechanics Reviews (ASME), 60, pp. 195-216, 2007. http://dx.doi.org/10.1115/1.2777164

[2] Daniel, I.M. \& Ishai, O., Engineering Mechanics of Composite Materials, Oxford University Press: New York, 2006.

[3] Sladek, V., Sladek, J. \& Sator, L., Physical decomposition of thin plate bending problems and their solution by mesh-free methods. Engineering Analysis with Boundary Elements, 37, pp. 348-365, 2013. http://dx.doi.org/10.1016/j.enganabound.2012.11.005

[4] Sator, L., Sladek, V. \& Sladek, J., Coupling effects in elastic analysis of FGM composite plates by mesh-free methods. Composite Structures, 115, pp. 100-110, 2014. http://dx.doi.org/10.1016/j.compstruct.2014.04.016

[5] Sladek, V., Sladek, J. \& Sator, L., Mesh-free formulations for solution of bending problems for thin elastic plates with variable bending stiffness. Part I: mathematical formulation. Building Research Journal, 60, pp. 111-148, 2012.

[6] Sator, L., Sladek, V. \& Sladek, J., Mesh-free formulations for solution of bending problems for thin elastic plates with variable bending stiffness. Part II: numerical solutions. Building Research Journal, 60, pp. 149-170, 2012.

[7] Sator, L., Sladek, V., Sladek, J. \& Young, D.L., Elastodynamics of FGM plates by meshfree method. Composite Structures, 140, pp. 309-322, 2016.

http://dx.doi.org/10.1016/j.compstruct.2015.12.065 
[8] Wilson, E.L., Farhoomand, I. \& Bathe, K.J., Nonlinear dynamic analysis of complex structures. Earthquake Engineering \& Structural Dynamics, 1, pp. 241-252, 1973. http://dx.doi.org/10.1002/eqe.4290010305

[9] Lancaster, P. \& Salkauskas, K., Surfaces generated by moving least square method. Mathematics of Computation, 37, pp. 141-158, 1981. http://dx.doi.org/10.1090/S0025-5718-1981-0616367-1

[10] Sladek, V., Sladek, J. \& Zhang, C., Computation of stresses in non-homogeneous elastic solids by local integral equation method: a comparative study. Computational Mechanics, 41, pp. 827-845, 2008. http://dx.doi.org/10.1007/s00466-006-0155-5

[11] Sladek, V., Sladek, J. \& Zhang, C., Local integral equation formulation for axially symmetric problems involving elastic FGM. Engineering Analysis with Boundary Elements, 32, pp. 1012-1024, 2008.

http://dx.doi.org/10.1016/j.enganabound.2007.09.006

[12] Sladek, V., Sladek, J. \& Zhang, C., On increasing computational efficiency of local integral equation method combined with meshless implementations. Computer Modeling in Engineering \& Sciences, 63, pp. 243-263, 2010.

[13] Sladek, V. \& Sladek, J., Local integral equations implemented by MLS-approximation and analytical integrations. Engineering Analysis with Boundary Elements, 34, pp. 904-913, 2010.

http://dx.doi.org/10.1016/j.enganabound.2010.03.015

[14] Gladwell, I. \& Thomas, R., Stability of the Newmark, Houbolt and Wilson $\theta$ methods. International Journal for Numerical and Analytical Methods in Geomechanics, 4, pp. 143-158, 1980.

http://dx.doi.org/10.1002/nag.1610040205

[15] Hughes, T.J.R., The Finite Element Method, Prentice-Hall Inc: New Jersey, 1987. 